\title{
Selection of Indexing Terms by Authors: A Proposal for Abstracts Submitted to the 1985 Meetings of the APS-SPR-APA
}

\author{
REX G. BICKERS
}

Children's Hospital, Ohio State University, Columbus, Ohio 43205

The practice of indexing and cataloging written documents is as ancient as the great scroll libraries of Ptolemy in Alexandria. In modern times, however, the task of indexing the biomedical and scientific literature has taken on enormous proportions. Over 20 years ago, it was widely believed that the literature explosion had reached a crisis stage; thus, between 1960 and 1966, a new set of computer-based solutions was sought. In response, the United States National Library of Medicine began development of what we now know as MEDLINE (Medical Literature Analysis and Retrieval System, On Line). This system has become the prototype and world standard in the management of indexing and computer storage of bibliographic information.

Even MEDLINE has its limitations. Abstracts which appear in journals as part of a meeting program are not indexed in MEDLINE, or in its print version, Index Medicus. The relative merits of scientific work published in abstract form only (versus work later published as complete manuscripts) is a subject of some controversy $(1,2)$. Nevertheless, as publication lag time increases, abstracts are taking on increasing importance as a source of information on new areas of original investigation and clinical observations. The program issues of various scholarly journals, including Pediatric Research, are often the only source of certain referenced citations for support of grant proposals, for background in subsequent investigations, or for use in education.

For 1983 and 1984, the American Pediatric Society and the Society for Pediatric Research added a key word index to their annual program issue (of over 500 pages) to help readers locate related abstracts. Authors were asked to submit three key word terms used to generate the index. Although useful, the key word index is rather unwieldy to use and lacks cross-referencing to a standardized medical vocabulary. As a result, an experimental microcomputer-based system was devised as an alternative for the 1984 meetings. The system contained information on all 773 presentation abstracts and provided electronic search and retrieval capability based on any abstract title word as well as author-selected key words. The retrieval included presentation room, date, and time information which could ordinarily be found only in a separately located program listing.

To enhance this computer system at the 1985 meetings, a proposal has been adopted to require the use of standardized index terms. These terms must be selected from the Medical Subject Headings (MeSH) vocabulary of Index Medicus. Authors will select $\mathrm{MeSH}$ terms based on their own interpretation of the key concepts in the abstract. An index term selection form and instructions for its completion will accompany the abstract submission forms for 1985 .

Index terms will be selected in two steps, in a pattern similar to the standard MEDLINE indexing process, reviewed in detail elsewhere (3). The first and simplest form of $\mathrm{MeSH}$ indexing is the application of check tags; these are broad general terms which describe characteristics of study subjects or study design. The index term selection form will offer a modified list of check tags under the heading "checklist terms." This will provide authors an opportunity to list study subjects' age, sex, animal species (or human), and type of report without using up one of the three choices for more specific key word index terms.

The second step is the selection of three specific index terms from the MeSH vocabulary. Not all investigators and authors will be expected to be thoroughly familiar with details of the $\mathrm{MeSH}$ vocabulary, and most will find that it takes some effort to consider the most important indexable aspects of an individual abstract (or article). Indexing is a specific skill which combines subject knowledge, analytical thinking, and art.

$\mathrm{MeSH}$ descriptors are cataloged in a three-volume set of Medical Subject Headings, published as an accompaniment to Index Medicus and MEDLINE. The three volumes are entitled: $A n$ notated Alphabetic List, Tree Structures, and Permuted MeSH. Each represents a different system of organization of the MeSH vocabulary system. It should be remembered that the biomedical librarian is a specialist in the use of these materials. Thus, a librarian can be an author's most important resource for increasing his familiarity with Index Medicus and other references.

The maintenance of the MeSH vocabulary is a dynamic process. Continuous review provides for revision or addition of new terms, but the process is not perfect. One anticipated offshoot of this proposal is that authors will collectively create an inventory of $\mathrm{MeSH}$ deficiencies and inaccuracies in areas of research related to pediatrics. Authors of abstracts submitted to the APS-SPRAPA meetings, taken as an aggregate, are uniquely qualified to advise the National Library of Medicine on new trends and terminology in pediatrics.

The creation of a searchable computerized abstract database with a standardized vocabulary of index terms can be a valuable and innovative resource. It will improve the quality of the printed meeting program and facilitate its preparation. It also will provide unique possibilities for information retrieval before, during, and after the meeting. The support of all abstract contributors as well as the membership-at-large will ensure its success.

Acknowledgments. The author is grateful for guidance and support from Dr. William Berman, Jr. (Society for Pediatric Research) and Mr. Dewey Sehring (Ross Laboratories) in the initiation and development of this endeavor.

\section{REFERENCES}

1. Goldman, L, Loscalzo A 1980 Fate of cardiology research originally published in abstract form. N Engl J Med 303:255-259

2. Meranze J, Ellison N, Greenhow DE 1982 Publications resulting from anesthesia meeting abstracts. Anesth Analg 61:445-448

3. Bachrach CA, Charen T 1978 Selection of MEDLINE contents, the development of its thesaurus, and the indexing process. Med Inform 3:237-254

Reprint requests should be addressed to Rex G. Bickers, Children's Hospital, 700 Children's Drive, Columbus, OH 43205. 